
\title{
PReserch Square \\ Predictors of life satisfaction and healthy aging in Brunei: A gender comparative study
}

Hanif Abdul Rahman ( $\square$ hanif.rahman@ubd.edu.bn )

Universiti Brunei Darussalam

Armah Tengah

Universiti Brunei Darussalam

Yusnani Mohd Yusof

Universiti Brunei Darussalam

Ly Slesman

Universiti Brunei Darussalam

Chang-Yau Hoon

Universiti Brunei Darussalam

Khadizah H. Abdul-Mumin

Universiti Brunei Darussalam

\section{Research Article}

Keywords: Gender, Healthy aging, Life satisfaction, Brunei, Predictors, Multivariate analysis

Posted Date: July 7th, 2021

DOl: https://doi.org/10.21203/rs.3.rs-668936/v1

License: (c) (i) This work is licensed under a Creative Commons Attribution 4.0 International License.

Read Full License 


\section{Abstract \\ Objective}

Research on aging has received considerable attention in medicine, public health and social sciences in recent decades. This study aimed to investigate predictors of life satisfaction and healthy aging with focus on gender differences among elderly population in Brunei.

\section{Methods}

Cross-sectional study on elderly population aged 50 to 75 , recruited by proportionate sampling. Multivariate linear regression analysis stratified by gender, was applied.

\section{Results}

$45.6 \%$ of the variance explaining life satisfaction of elderly women were strongly associated with selfperceived health, social relationship, and education level. For elderly men, $26.3 \%$ of the variance of life satisfaction was predicted by physical functioning or disability, and social relationship. Musculoskeletal discomfort, and mental and emotional issues were significantly higher in elderly women.

\section{Conclusions}

This report benefits policymakers and related stakeholders for care of elderly by maintaining or further promoting social interactions, active engaging elderly in health maintenance, and physical and mental functioning.

\section{Introduction}

The world is rapidly aging as increasing longevity and decreasing fertility has become demographic trends $(1,2)$. Research on aging has received considerable attention in medicine, public health and social sciences in recent decades, however, fewer had approached the importance of gender for successful aging (3). It has become increasingly important to have gender-specific approaches to understand gender gaps in the elderly population. Prior evidences have suggested strong differences in disease distribution, clinical profiles, and socioeconomic status, among elderly men and women (4-7). Aging population with higher chronic illnesses, disabilities, and dependency have significant economic and societal impact that will result in the rise of global burden of disease and disability $(2,8,9)$. There has been paramount efforts by government actors in collaboration with related stakeholders to increase period of 'good health' and sustained sense of well-being, to extend social productivity of elderly population, as much as possible (10). 
Life satisfaction, a subjective feeling and attitude about one's life at a particular point of time that could range from positive to negative, is one of the most important component of healthy aging (11). Life satisfaction elicits a U-shaped pattern with age, meaning as a person grow older their overall satisfaction in life rises after a nadir at 50 years (12). This corroborates with similar studies which demonstrated increasing life satisfaction with ageing (13-15) until it reaches a threshold at a certain point, which could differ by multitude of factors, and starts to decline $(16,17)$.

Successful aging is largely measured by subjective well-being, where life satisfaction is the major indicator (7). There has been numerous studies investigating significant predictors of life satisfaction, however, gender-specific studies are scarce, which withhold benefit of enriching discussion and thus understanding of well-being and its determinants $(7,18-21)$. Furthermore, prior evidence has established that healthy aging, is a strong contributor towards positive life satisfaction, that affect elderly functioning and the primary focus current gerontological effort to achieve more positive outcome of aging (7).

Since the early 20th century, life expectancy has increased substantially and along with the concomitant decline in fertility rate, an ageing population is on the rise around the world, and Brunei is no exception. A country is considered as an ageing population when 7 percent of the population are 65 years and above. According to the United Nation, Brunei's older population will account for 7.7 per cent of the total population by 2025 (22). This means in no time Brunei will have relatively higher proportion of its inhabitants being elderly compared to children (22). By 2050, global life expectancy was estimated to increase by 4.5 years, an approximate six percent rise since 2015, and was predicted to increase continuously thereafter (22). Still, questions remain as to whether these added years are experienced in good health and life satisfaction.

Therefore, this study was conducted with the purpose of investigating the predictors of life satisfaction and healthy aging with focus on gender differences.

\section{Methods}

\section{Study design}

A cross-sectional study using interviewer-assisted questionnaire administration that was disseminated nationwide in all four districts of Brunei Darussalam.

\section{Participant selection and setting}

The study targeted participants who were within the age range of 50 to 75 years; held Brunei citizenship or permanent residents; lived at home or resided in an institution; lived in a household; oriented to place and persons and able to communicate in English and Malay. Adults aged 50 years or below and those who were cognitively impaired were excluded. The survey was conducted at the home of respondents.

The Ministry of Home Affairs via the District offices granted permission to access the current population census of the four districts in Brunei. All eligible participants $(n=63,900)$ were then sectioned out from 
the total study population $(n=76,000)$. Minimum sample size was calculated with precision of $5 \%(d=$ 0.05). A minimum sample of 385 was required to achieve precision of 0.5 at $95 \%$ confidence interval of population size of 37,917 . Stratified proportionate sampling was employed to ensure recruitment of participants were proportional to district and gender of study population.

\section{Data collection instruments}

Data was collected by appointed research assistants whom were trained prior to data collections using a questionnaire adapted and developed from various validated and established tools including:

1. Health and Retirement Study on Psychosocial and Lifestyle developed in the University of Michigan, United States (HRS);

2. English Longitudinal Survey on Aging (ELSA) jointly run by teams at University College London $(\mathrm{UCL})$, the Institute for Fiscal Studies (IFS), NatCen Social Research and the University of Manchester in United Kingdom;

3. Survey on Health, Aging and Retirement in Europe (SHARE);

4. Irish Longitudinal Study on Aging (TILDA) developed in Trinity College Dublin;

5. Korean Longitudinal Study of Aging (KLoSA);

6. Japanese Study of Aging and Retirement (JSTAR).

A panel was formed to review the questions in the six questionnaires and decided to extract only relevant questions in the context of Brunei. The panel was comprised of ten experts from different disciplines (Health, Economics, Business, Psychology, Social Studies, and Policy). All the relevant questions were compiled as one questionnaire that consists of: demographics, family, health, income, and life satisfactions.

The questionnaire was pre-tested on twenty elderly participants with the same inclusion criteria. Amendments to the questionnaire were made based on the feedback and comments given by the participants. In the main study, two research assistants accompanied by a member of the research team collected the data. Participants were able to ask questions shall they require assistance. Full explanation of the study was offered and any questions regarding the study was answered prior to completing the questionnaire.

\section{Data analysis}

All analyses were stratified by elderly men and women. Descriptive statistics were used to describe the sample. Univariate analysis using Chi-square test for independence and Independent $t$ test were applied, where indicated. Multiple linear regression analyses were then performed for using stepwise automatic variable selection procedure. The factors were selected based on the best model for each outcome. The significant factors were then checked for interaction effects. Variance inflation factor (VIF) was used to check for multicollinearity. Residual plots were used to check for assumption for overall linearity, linearity of each numerical independent variable, normality, and equal variance. 'Standardized' residual plots were 
used to check for outliers. All statistical analyses were performed using RStudio v1.1.383. All statistical tests are two-sided and a $P$ value $<0.05$ is considered statistically significant.

\section{Ethical considerations}

The research protocol of this study has been approved by the research ethics committee of Universiti Brunei Darussalam (UBD/OAVCR/UREC/Apr18-04). This study conforms with ethical standards to ensure safeguarding and protection human rights were sufficient according to declaration of Helsinki (23). Written informed consent was obtained prior to the study.

\section{Results}

In total, 429 elderly persons participated in the study. Table 1 presents characteristics of the sample segregated by gender where $50.1 \%$ were male and $49.9 \%$ were female. A significant portion of the sample were recruited from Brunei-Muara district $(77.4 \%, p=0.029)$. Majority (about $94 \%$ ) of the sample were of ethic Malay origin and of Islamic belief (96\%). One in four (about $24 \%$ ) of the participants were membership of an organisation, clubs or societies where they mostly had activity 2 to 3 times every week (male $=35.9 \%$ and female $64.1 \%$ ). 
Table 1

Descriptive statistics of participants' characteristics by gender $(n=429)$

\begin{tabular}{|c|c|c|c|c|c|}
\hline & \multicolumn{2}{|c|}{ Male $(n=215)$} & \multicolumn{2}{|c|}{ Female $(n=214)$} & \multirow[b]{2}{*}{ P-value ${ }^{a}$} \\
\hline & $\mathrm{n}$ & (\%) & $\mathrm{n}$ & (\%) & \\
\hline Mean Age of participants in Years (SD) & 60.1 & $(5.7)$ & 60.9 & $(6.1)$ & $0.144^{b}$ \\
\hline Mean Age of spouse in Years (SD) & 57.8 & $(6.5)$ & 63.1 & $(7.0)$ & $<0.001^{b}$ \\
\hline \multicolumn{6}{|l|}{ Marital status } \\
\hline Married & 195 & $(57.9)$ & 142 & $(42.1)$ & $<0.001$ \\
\hline Separated & 2 & $(50.0)$ & 2 & $(50.0)$ & \\
\hline Divorced & 4 & $(30.8)$ & 9 & $(69.2)$ & \\
\hline Widowed & 8 & $(18.2)$ & 36 & $(81.8)$ & \\
\hline Single & 6 & $(19.4)$ & 25 & $(80.6)$ & \\
\hline \multicolumn{6}{|l|}{ Living together with spouse? } \\
\hline Each wife/husband living individual household & 16 & $(66.7)$ & 8 & $(33.3)$ & $<0.001$ \\
\hline All wives/husband living under one household & 142 & $(78.0)$ & 40 & $(22.0)$ & \\
\hline Not specified or responded or refused & 57 & $(25.7)$ & 165 & $(74.3)$ & \\
\hline \multicolumn{6}{|l|}{ Living together with household members? } \\
\hline Yes & 161 & $(48.6))$ & 170 & $(51.4)$ & 0.080 \\
\hline \multicolumn{6}{|l|}{ How many children live together with you? } \\
\hline None & 21 & $(35.6)$ & 38 & $(64.4)$ & 0.018 \\
\hline 1 to 2 children & 61 & $(45.2)$ & 74 & $(54.8)$ & \\
\hline 3 to 4 children & 73 & $(53.3)$ & 64 & $(46.7)$ & \\
\hline 5 to 6 children & 50 & $(62.5)$ & 30 & $(37.5)$ & \\
\hline$>6$ children & 10 & $(55.6)$ & 8 & $(44.4)$ & \\
\hline \multicolumn{6}{|l|}{ Highest level of education attained } \\
\hline No formal education & 1 & $(4.2)$ & 23 & $(95.8)$ & $<0.001$ \\
\hline Primary school & 10 & $(28.6)$ & 25 & $(71.4)$ & \\
\hline Lower secondary (Form 1-3) & 58 & $(51.8)$ & 54 & $(48.2)$ & \\
\hline Upper Secondary (Form 4-5) & 49 & $(43.0)$ & 65 & $(57.0)$ & \\
\hline
\end{tabular}




\begin{tabular}{|c|c|c|c|c|c|}
\hline \multirow[b]{2}{*}{ Form 6} & \multicolumn{2}{|c|}{ Male $(n=215)$} & \multicolumn{3}{|c|}{ Female $(n=214)$} \\
\hline & 24 & $(68.6)$ & 11 & $(31.4)$ & \\
\hline Diploma & 16 & $(61.6)$ & 10 & $(38.5)$ & \\
\hline Higher National Diploma & 20 & $(74.1)$ & 7 & $(25.9)$ & \\
\hline Bachelor Degree & 24 & $(66.7)$ & 12 & $(33.3)$ & \\
\hline Postgraduate Degree & 13 & $(65.0)$ & 7 & $(35.0)$ & \\
\hline \multicolumn{6}{|l|}{ Estimated income (B\$) } \\
\hline 250 and below & 25 & $(22.9)$ & 84 & $(77.1)$ & $<0.001$ \\
\hline 251 to 500 & 15 & $(39.5)$ & 23 & $(60.5)$ & \\
\hline 501 to 1000 & 32 & $(60.4)$ & 21 & $(39.6)$ & \\
\hline 1001 to 1500 & 53 & $(57.6)$ & 39 & $(42.4)$ & \\
\hline 1501 to 2000 & 31 & $(68.9)$ & 14 & $(31.1)$ & \\
\hline 2001 to 2500 & 22 & $(59.5)$ & 15 & $(40.5)$ & \\
\hline 2501 to 3000 & 17 & $(60.7)$ & 11 & $(39.3)$ & \\
\hline 3001 to 3500 & 11 & $(78.6)$ & 3 & $(21.4)$ & \\
\hline 3501 and above & 9 & $(69.2)$ & 4 & $(30.8)$ & \\
\hline
\end{tabular}

Univariate analysis showed that male participants were significantly higher in terms of smoking, married status, and living with spouse compared to female participants $(p<0.001)$. Female participants had significantly higher in terms of having 1 to 2 children. On the other hand, male participants had significantly higher in terms of having 3 or more children $(p=0.018)$. Male participants also had significantly higher educational attainment where it was observed that proportion of male was higher from Form 6 (pre-university) and above $(p<0.001)$. Male participants also had significantly higher estimated income where it was observed that proportion of male was higher from $\mathrm{B} \$ 501$ and above $(p<$ $0.001)$.

Table 2 illustrates the health perception, life satisfaction, and ability to do daily activities. Univariate analysis showed that elderly men $(60.6 \%)$ had significantly higher perception of "very good" health compared to elderly women $(39.4 \%)(p<0.001)$. This is similar for life satisfaction where $79.0 \%$ of elderly men and $65.4 \%$ of women reported "satisfied" with life. Nonetheless, both gender were equally concern regarding importance of health $(p=0.740)$ where more than $80 \%$ had undergone health screening or medical checks. 
Table 2

Health perception, Satisfaction with life and daily activities of participants by gender $(n=429)$

\begin{tabular}{|c|c|}
\hline Male $(n=215)$ & $\begin{array}{l}\text { Female }(n= \\
214)\end{array}$ \\
\hline n $\quad(\%)$ & (\%) \\
\hline
\end{tabular}

Overall current health

Very good

$\begin{array}{lllll}83 & (60.6) & 54 & (39.4) & 0.001 \\ 95 & (50.5) & 93 & (49.5) & \\ 30 & (33.3) & 60 & (66.7) \\ 7 & (50.0) & 7 & (50.0) & \end{array}$

Good

$\begin{array}{lllll}170 & (54.8) & 140 & (45.2) & 0.008\end{array}$

Satisfied

$37 \quad(42.0) \quad 51 \quad(58.0)$

Fairly satisfied

$5 \quad(29.4) \quad 12 \quad(70.6)$

Somewhat satisfied

$2 \quad(40.0) \quad 3$

$(60.0)$

Unsatisfied

1

$(11.1)$

(88.9)

\section{Performing everyday activities}

Difficulty using any vehicles by yourself

Difficulty to shop for daily needs

Difficulty to shower on your own

Difficulty to go out and pay bills

Difficulty to make deposits and withdrawal from bank

Difficulty to collect your pension and so on

Difficulty to read any

newspapers/books/magazines

Interested in articles or programs about health

Do you visit the homes of friends

Do you give advice to family or friends

Can you visit sick people?

Do you ever talk to young people?

$195 \quad(57.7) \quad 143 \quad(42.3) \quad<0.001$

$\begin{array}{lllll}199 & (50.9) & 192 & (49.1) & 0.387\end{array}$

$\begin{array}{lllll} & 198 \quad(49.0) & 206 & (51.0) & 0.102\end{array}$

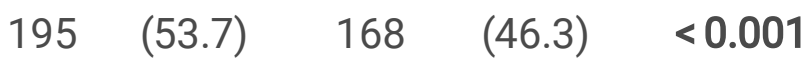

$\begin{array}{lllll} & 191 & (54.7) & 158 & (45.3)\end{array}$

$\begin{array}{lllll}171 & \text { (55.5) } & 137 & \text { (44.5) } & 0.001\end{array}$

$\begin{array}{lllll}180 & (50.0) & 180 & (50.0) & 1.000\end{array}$

$178 \quad(50.0) \quad 178 \quad(50.0) \quad 1.000$

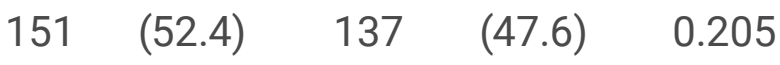

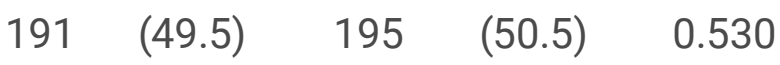

$\begin{array}{lllll} & 198 & \text { (49.9) } & 199 & 0.865\end{array}$

$\begin{array}{lllll}190 & (49.9) & 191 & (50.1) & 0.892\end{array}$




\begin{tabular}{|llllll|}
\hline & Male $(\boldsymbol{n = 2 1 5})$ & \multicolumn{3}{l}{ Female $(\boldsymbol{n}=$} \\
\hline & 195 & $(50.9)$ & 188 & $(49.1)$ & 0.425 \\
\hline Do you use telephone by yourself? & 192 & $(50.4)$ & 179 & $(49.6)$ & 0.878 \\
\hline Do you take medicine by yourself? & 193 & $(60.5)$ & 126 & $(39.5)$ & $<0.001$ \\
\hline Do you drive? & 190 & $(47.9)$ & 207 & $(52.1)$ & 0.002 \\
\hline Can you prepare your own meals? & 150 & $(51.2)$ & 143 & $(48.8)$ & 0.581 \\
\hline Do you do gardening/fishing/or other hobbies? & & & & & \\
\hline Walking in the course of daily activities & 9 & $(36.0)$ & 16 & $(64.0)$ & 0.106 \\
\hline Hardly walk & 28 & $(43.8)$ & 36 & $(56.2)$ & \\
\hline Less than 30 minutes & 69 & $(47.9)$ & 75 & $(52.1)$ & \\
\hline 30-60 minutes & 26 & $(49.1)$ & 27 & $(50.9)$ & \\
\hline $61-90$ minutes & 80 & $(57.1)$ & 60 & $(42.9)$ & \\
\hline More than 90 minutes & 3 & $(100.0)$ & 0 & $(0.0)$ & \\
\hline Cannot walk & & & & & \\
\hline n = count/frequency & & & & & \\
\hline a = Chi-square test for independence & & & & \\
\hline
\end{tabular}

In terms of performing daily activities, it was reported that current health problems did not impair physical functioning. Significantly higher male sample (61.8\%) compared to female sample (38.2\%) still drive by themselves although they also had significantly higher report of difficulty when using vehicles $(p<0.001)$. They also reported significantly higher difficulty to go out and pay bills, make withdrawals or deposits, and collect pensions. On the other hand, female participants reported significantly higher difficulty in preparing meals for themselves.

Table 3 presents the mental and emotional conditions of participants last week. The stratified analysis by gender revealed that emotional conditions among the elderly population were similar, in general. In conditions where there were significant differences, female participants exhibited higher emotional problems for the past week, particularly in: 'feeling frightened', 'feeling lonely', 'feel like crying', 'feeling sad', 'difficulty concentrating what I was doing', and 'something normally effortless became difficult to do'. 
Table 3

Mental and Emotional conditions of participants last week by gender $(n=429)$.

\begin{tabular}{lllll}
$\begin{array}{l}\text { Male }(n= \\
\text { 215) }\end{array}$ & \multicolumn{2}{l}{ Female $(n=$} \\
$\mathrm{n}$ & $(\%)$ & $\mathrm{n}$ & $(\%)$ & $\begin{array}{l}\text { P-value } \\
\mathrm{a}\end{array}$
\end{tabular}

\section{Emotional condition last week}

1. Felt depressed

$\begin{array}{llllll}\text { Not at all } & 172 & (53.1) & 152 & (46.9) & 0.083 \\ \text { Sometimes } & 32 & (42.7) & 43 & (57.3) & \\ \text { Always } & 3 & (23.1) & 10 & (76.9) & \\ \text { Not applicable } & 8 & (47.1) & 9 & (52.9)\end{array}$

2. Felt could not do a normal person could do

$\begin{array}{llllll}\text { Not at all } & 30 & (60.0) & 20 & (40.0) & 0.282 \\ \text { Sometimes } & 28 & (41.8) & 39 & (58.2) & \\ \text { Always } & 147 & (50.3) & 145 & (49.7) & \\ \text { Not applicable } & 10 & (50.0) & 10 & (50.0)\end{array}$

3. Could not concentrate what I was doing

$\begin{array}{llllll}\text { Not at all } & 147 & (56.5) & 113 & (43.5) & 0.012 \\ \text { Sometimes } & 29 & (41.4) & 41 & (58.6) & \\ \text { Always } & 31 & (39.2) & 48 & (60.8) & \\ \text { Not applicable } & 8 & (40.0) & 12 & (60.0)\end{array}$

4. Something that is normally effortless was difficult to do

Not at all

Sometimes

Always

Not applicable

5. Felt the future is bright

Not at all

Sometimes $\begin{array}{lllll}144 & (56.3) & 112 & (43.8) & 0.017\end{array}$

$\begin{array}{llll}46 \quad(43.0) & 61 & (57.0)\end{array}$

$17 \quad(36.2) \quad 30 \quad(63.8)$

$8 \quad(42.1) \quad 11 \quad$ (57.9)

$\begin{array}{lllll}26 & (61.9) & 16 & (38.1) & 0.334 \\ 38 & (47.5) & 42 & (52.5) & \end{array}$




\begin{tabular}{|c|c|c|c|c|c|}
\hline \multirow[b]{2}{*}{ Always } & \multicolumn{2}{|c|}{$\begin{array}{l}\text { Male }(n= \\
215)\end{array}$} & \multicolumn{2}{|c|}{$\begin{array}{l}\text { Female }(n= \\
214)\end{array}$} & \\
\hline & 124 & $(50.4)$ & 122 & $(49.6)$ & \\
\hline Not applicable & 27 & $(44.3)$ & 34 & $(55.7)$ & \\
\hline \multicolumn{6}{|c|}{ 6. Felt life so far has been a failure } \\
\hline Not at all & 174 & (51.5) & 164 & $(48.5)$ & 0.606 \\
\hline Sometimes & 22 & $(47.8)$ & 24 & (52.2) & \\
\hline Always & 2 & $(40.0)$ & 3 & $(60.0)$ & \\
\hline Not applicable & 16 & $(41.0)$ & 23 & $(59.0)$ & \\
\hline \multicolumn{6}{|c|}{ 7. Felt frightened } \\
\hline Not at all & 159 & (54.3) & 134 & $(45.7)$ & 0.018 \\
\hline Sometimes & 43 & $(39.4)$ & 66 & $(60.6)$ & \\
\hline Always & 1 & (16.7) & 5 & (83.3) & \\
\hline Not applicable & 12 & $(57.1)$ & 9 & (42.9) & \\
\hline \multicolumn{6}{|c|}{ 8. Could not sleep well } \\
\hline Not at all & 133 & $(55.6)$ & 106 & (44.4) & 0.062 \\
\hline Sometimes & 60 & $(45.1)$ & 73 & $(54.9)$ & \\
\hline Always & 19 & (38.0) & 31 & $(62.0)$ & \\
\hline Not applicable & 3 & (42.9) & 4 & $(57.1)$ & \\
\hline \multicolumn{6}{|l|}{ 9. Felt happy } \\
\hline Not at all & 20 & $(45.5)$ & 24 & (54.5) & 0.827 \\
\hline Sometimes & 19 & $(46.3)$ & 22 & $(53.7)$ & \\
\hline Always & 165 & (50.9) & 159 & $(49.1)$ & \\
\hline Not applicable & 11 & $(55.0)$ & 9 & (45.0) & \\
\hline \multicolumn{6}{|c|}{ 10. Felt more reserved than usual } \\
\hline Not at all & 148 & (50.5) & 145 & $(49.5)$ & 0.981 \\
\hline Sometimes & 48 & $(49.0)$ & 50 & $(51.0)$ & \\
\hline Always & 7 & $(46.7)$ & 8 & (53.3) & \\
\hline Not applicable & 12 & $(52.2)$ & 11 & $(47.8)$ & \\
\hline
\end{tabular}




Male
$215)$$(n=\quad$ Female $(n=$

11. Felt lonely

$\begin{array}{llllll}\text { Not at all } & 157 & (57.3) & 117 & (42.7) & <0.001 \\ \text { Sometimes } & 46 & (37.7) & 76 & (62.3) \\ \text { Always } & 4 & (23.5) & 13 & (76.5) \\ \text { Not applicable } & 8 & (50.0) & 8 & (50.0)\end{array}$

12. People around me seem cold to me

\begin{tabular}{llllll} 
Not at all & 179 & $(51.7)$ & 167 & $(48.3)$ & 0.226 \\
Sometimes & 19 & $(39.6)$ & 29 & $(60.4)$ \\
\hline Always & 5 & $(71.4)$ & 2 & $(28.6)$ \\
\hline Not applicable & 12 & $(42.9)$ & 16 & $(57.1)$
\end{tabular}

13. Cried or felt like crying

\begin{tabular}{lllllll|} 
Not at all & 181 & $(57.8)$ & 132 & $(42.2)$ & $<0.001$ \\
Sometimes & 21 & $(23.9)$ & 67 & $(76.1)$ & \\
Always & 2 & $(33.3)$ & 4 & $(66.7)$ & \\
Not applicable & 11 & $(50.0)$ & 11 & $(50.0)$ & \\
14. Felt sad & & & & & & $<0.001$ \\
Not at all & 162 & $(57.4)$ & 120 & $(42.6)$ & $<$ \\
Sometimes & 39 & $(32.0)$ & 83 & $(68.0)$ & \\
Always & 2 & $(40.0)$ & 3 & $(60.0)$ & \\
Not applicable & 12 & $(60.0)$ & 8 & $(40.0)$ & \\
15. Felt people around me disliked me & & & & & \\
Not at all & 182 & $(52.1)$ & 167 & $(47.9)$ & 0.254 \\
\hline Sometimes & 19 & $(43.2)$ & 25 & $(56.8)$ & \\
\hline Always & 1 & $(20.0)$ & 4 & $(80.0)$ & \\
\hline Not applicable & 13 & $(42.0)$ & 18 & $(58.0)$ & \\
\hline 16. Didn't feel like doing anything & & & & & \\
\hline Not at all & 152 & $(52.8)$ & 136 & $(47.2)$ & 0.223 \\
\hline
\end{tabular}




\begin{tabular}{|llllll|}
\hline & \multicolumn{3}{l}{ Male $(\boldsymbol{n}=$} & \multicolumn{3}{l|}{ Female $(\boldsymbol{n}=$} \\
\hline Sometimes & 215) & & 214) \\
\hline Always & 44 & $(42.7)$ & 59 & $(57.3)$ \\
\hline Not applicable & 6 & $(66.7)$ & 3 & $(33.3)$ \\
\hline $\mathrm{n}=$ count/frequency & 13 & $(44.8)$ & 16 & $(55.2)$ \\
$\mathrm{a}=$ Chi-square test for independence & & & & \\
\hline
\end{tabular}

Table 4 illustrates the social health of the sample. Univariate analysis showed that the spouse of male participants $(61.0 \%)$ would be significantly more concern about them compared to spouse of female participants $(39.0 \%)(p<0.001)$. Similarly, male participants $(59.4 \%)$ were significantly more concerned about their spouse if they had problem compared to female participants $(40.6 \%)(p<0.001)$. Both male and female participants reported visiting friends or relatives equally frequent, at least once a month. In addition, most of them had more than one close friends or relatives that they could confide and call for help. 
Table 4

Social health of elderly persons by gender $(n=429)$.

\begin{tabular}{|c|c|c|c|c|c|}
\hline \multirow[b]{2}{*}{ Social Health } & \multicolumn{2}{|c|}{$\begin{array}{l}\text { Male }(n= \\
215)\end{array}$} & \multicolumn{2}{|c|}{$\begin{array}{l}\text { Female }(n= \\
214)\end{array}$} & \multirow[b]{2}{*}{$\begin{array}{l}\text { P-value } \\
\text { a }\end{array}$} \\
\hline & $\mathrm{n}$ & $(\%)$ & $\mathrm{n}$ & $(\%)$ & \\
\hline \multicolumn{6}{|c|}{ Frequency of meeting relatives or friends } \\
\hline Almost every day & 77 & $(58.8)$ & 54 & $(41.2)$ & 0.090 \\
\hline 2-3 times a week & 54 & $(50.0)$ & 54 & $(50.0)$ & \\
\hline Once a week & 23 & $(56.1)$ & 18 & $(43.9)$ & \\
\hline Every two weeks & 27 & $(42.9)$ & 36 & $(57.1)$ & \\
\hline Once a month & 12 & $(32.4)$ & 25 & $(67.6)$ & \\
\hline Every two months & 3 & $(27.2)$ & 8 & $(72.7)$ & \\
\hline Three or four times a year & 2 & $(33.3)$ & 4 & $(66.7)$ & \\
\hline Once or twice a year & 7 & $(46.7)$ & 8 & $(53.3)$ & \\
\hline No close friends or relatives & 2 & $(40.0)$ & 3 & $(60.0)$ & \\
\hline Refused or no answer & 8 & $(66.7)$ & 4 & $(33.3)$ & \\
\hline \multicolumn{6}{|c|}{$\begin{array}{l}\text { How many relatives you can talk at ease on private } \\
\text { matters }\end{array}$} \\
\hline None & 27 & $(58.7)$ & 19 & $(41.3)$ & 0.404 \\
\hline One person & 17 & $(47.2)$ & 19 & $(52.8)$ & \\
\hline Two persons & 31 & $(42.5)$ & 42 & $(57.5)$ & \\
\hline Three or four persons & 51 & $(49.0)$ & 53 & $(51.0)$ & \\
\hline Five to eight persons & 41 & $(47.7)$ & 45 & $(52.3)$ & \\
\hline Nine or more & 48 & $(57.1)$ & 36 & $(42.9)$ & \\
\hline \multicolumn{6}{|c|}{ How many close relatives you can call for help } \\
\hline None & 27 & $(54.0)$ & 23 & $(46.0)$ & 0.853 \\
\hline One person & 14 & $(41.2)$ & 20 & $(58.8)$ & \\
\hline Two persons & 35 & $(46.6)$ & 40 & $(53.3)$ & \\
\hline Three or four persons & 49 & $(52.1)$ & 45 & $(47.9)$ & \\
\hline Five to eight persons & 44 & $(51.2)$ & 42 & $(48.8)$ & \\
\hline
\end{tabular}




\begin{tabular}{|c|c|c|c|c|c|}
\hline \multirow[b]{2}{*}{ Nine or more } & \multicolumn{2}{|c|}{$\begin{array}{l}\text { Male }(n= \\
215)\end{array}$} & \multicolumn{2}{|c|}{$\begin{array}{l}\text { Female }(n= \\
214)\end{array}$} & \\
\hline & 46 & $(51.1)$ & 44 & $(48.9)$ & \\
\hline \multicolumn{6}{|c|}{ Who is primarily responsible for following tasks: } \\
\hline \multicolumn{6}{|l|}{ 1. Care of children } \\
\hline Self only & 10 & $(16.9)$ & 49 & $(83.1)$ & $<0.001$ \\
\hline Primarily self & 5 & $(21.7)$ & 18 & $(78.3)$ & \\
\hline Equally with spouse & 162 & $(61.1)$ & 103 & $(38.9)$ & \\
\hline Primarily spouse & 15 & $(78.9)$ & 4 & $(21.1)$ & \\
\hline Not applicable & 23 & $(36.5)$ & 40 & $(63.5)$ & \\
\hline \multicolumn{6}{|l|}{ 2. Earn income } \\
\hline Self only & 47 & $(49.5)$ & 48 & $(50.5)$ & $<0.001$ \\
\hline Primarily self & 17 & $(40.5)$ & 25 & $(59.5)$ & \\
\hline Equally with spouse & 131 & $(64.9)$ & 71 & $(35.1)$ & \\
\hline Primarily spouse & 12 & $(20.3)$ & 47 & $(79.7)$ & \\
\hline Not applicable & 8 & $(25.8)$ & 23 & $(74.2)$ & \\
\hline \multicolumn{6}{|l|}{ 3. Household chores } \\
\hline Self only & 19 & $(19.8)$ & 77 & $(80.2)$ & $<0.001$ \\
\hline Primarily self & 5 & $(10.4)$ & 43 & $(89.6)$ & \\
\hline Equally with spouse & 141 & $(65.6)$ & 74 & $(34.4)$ & \\
\hline Primarily spouse & 42 & $(93.3)$ & 3 & $(6.7)$ & \\
\hline Not applicable & 8 & $(32.0)$ & 17 & $(68.0)$ & \\
\hline \multicolumn{6}{|c|}{ 4. Care of older relatives } \\
\hline Self only & 11 & $(31.4)$ & 24 & $(68.6)$ & $<0.001$ \\
\hline Primarily self & 2 & $(28.6)$ & 5 & $(71.4)$ & \\
\hline Equally with spouse & 74 & $(67.3)$ & 36 & $(32.7)$ & \\
\hline Primarily spouse & 4 & $(50.0)$ & 4 & $(50.0)$ & \\
\hline Not applicable & 124 & $(46.3)$ & 144 & $(53.7)$ & \\
\hline
\end{tabular}




\begin{tabular}{|c|c|c|c|c|c|}
\hline \multirow[b]{2}{*}{ Self only } & \multicolumn{2}{|c|}{$\begin{array}{l}\text { Male }(n= \\
215)\end{array}$} & \multicolumn{2}{|c|}{$\begin{array}{l}\text { Female }(n= \\
214)\end{array}$} & \multirow[b]{2}{*}{$<0.001$} \\
\hline & 27 & (31.4) & 59 & $(68.6)$ & \\
\hline Primarily self & 8 & $(26.7)$ & 22 & (73.3) & \\
\hline Equally with spouse & 157 & $(61.3)$ & 99 & $(38.7)$ & \\
\hline Primarily spouse & 14 & $(50.0)$ & 14 & $(50.0)$ & \\
\hline Not applicable & 9 & $(31.0)$ & 20 & $(69.0)$ & \\
\hline \multicolumn{6}{|l|}{ 6. Decide on major purchases } \\
\hline Self only & 31 & (33.3) & 62 & $(66.7)$ & $<0.001$ \\
\hline Primarily self & 7 & $(20.6)$ & 27 & $(79.4)$ & \\
\hline Equally with spouse & 154 & $(62.1)$ & 94 & $(37.9)$ & \\
\hline Primarily spouse & 17 & $(60.7)$ & 11 & (39.3) & \\
\hline Not applicable & 6 & $(23.1)$ & 20 & $(76.9)$ & \\
\hline \multicolumn{6}{|l|}{$\mathrm{n}=$ count $/$ frequency } \\
\hline$a=$ Chi-square test for independence & & & & & \\
\hline
\end{tabular}

In terms of caring for children, in situations where the task was equally shared with spouse, male participants $(61.1 \%)$ reported significantly higher involvement compared to female participants (35.1\%). Conversely, in situations where the task of caring for children were done by self or primarily self only, female participants (78.3 to $83.1 \%$ ) reported significantly higher involvement compared to male participants (16.9-21.7\%). This was also consistent with further univariate analysis on other tasks including earning income, doing household chores, caring of older relatives, managing household accounts and deciding on major purchases.

Table 5 presents physical health status of the sample. The results showed that male and female participants were equally suffering from musculoskeletal pain. However, there was significantly higher reports of shoulder pain, back pain, leg pain and knee pain from female participants. It was also observed that female participants (51.7 to $70.7 \%$ ) received significantly higher health services such as home-based nursing and medical assistance devices (for example, wheelchair) compared to male participants (29.3 to 48.3\%). Participants were equally reporting issues with vision and hearing, kidney, liver and heart problems, high blood pressure, diabetes, and psychiatric disorders. 
Table 5

Physical health of elderly persons by gender $(n=429)$.

\section{Male $(n=215) \quad$ Female $(n=$ 214)}

\section{Physical health}

n (\%) n

(\%)

P-value

a

What part of body feel pain and how bad was

it?

1. Headache

Mild

$73 \quad(50.7) \quad 71$

(49.3)

0.198

Moderate

25

(37.3)

42

(62.7)

Severe

$1 \quad$ (33.3) 2

No pain

$97 \quad(53.9) \quad 83$

Not applicable

19

(54.3)

16

2. Shoulder

Mild

$38 \quad(41.8) \quad 53$

(58.2)

0.043

Moderate

$26 \quad$ (38.8) 41

Severe

$3 \quad(75.0) \quad 1$

No pain

$127 \quad$ (55.0)

20

(57.1) $\quad 15$

(61.2)

Not applicable

3. Arm

Mild

$27 \quad$ (40.3) 40

(59.7)

0.074

Moderate

22 (40.0)

33

(60.0)

Severe

2

No pain

140

(100.0)

0

(0.0)

Not applicable

24

(53.4)

122

(46.6)

4. Wrist

Mild

$26 \quad(44.1) \quad 33$

(55.9)

0.184

Moderate

$16 \quad$ (45.7) 19

Severe

$5 \quad(100.0) \quad 0$

No pain

$145 \quad(51.1) \quad 139 \quad$ (48.9)

(54.3) 


\begin{tabular}{|c|c|c|c|c|c|}
\hline \multirow[b]{2}{*}{ Not applicable } & \multicolumn{2}{|c|}{ Male $(n=215)$} & \multicolumn{2}{|c|}{$\begin{array}{l}\text { Female }(n= \\
214)\end{array}$} & \\
\hline & 23 & $(50.0)$ & 23 & $(50.0)$ & \\
\hline \multicolumn{6}{|l|}{ 5. Fingers } \\
\hline Mild & 19 & (37.3) & 32 & $(62.7)$ & 0.074 \\
\hline Moderate & 14 & $(37.8)$ & 23 & $(62.2)$ & \\
\hline Severe & 4 & $(80.0)$ & 1 & $(20.0)$ & \\
\hline No pain & 154 & $(53.1)$ & 136 & $(46.9)$ & \\
\hline Not applicable & 24 & $(52.2)$ & 22 & $(47.8)$ & \\
\hline \multicolumn{6}{|l|}{ 6. Chest } \\
\hline Mild & 25 & $(42.4)$ & 34 & $(57.6)$ & 0.502 \\
\hline Moderate & 14 & $(51.9)$ & 13 & $(48.1)$ & \\
\hline Severe & 4 & $(80.0)$ & 1 & $(20.0)$ & \\
\hline No pain & 147 & $(50.7)$ & 143 & $(49.3)$ & \\
\hline Not applicable & 25 & $(52.1)$ & 23 & $(47.9)$ & \\
\hline \multicolumn{6}{|l|}{ 7. Stomach ache } \\
\hline Mild & 30 & $(48.4)$ & 32 & $(51.6)$ & 0.757 \\
\hline Moderate & 17 & $(50.0)$ & 17 & $(50.0)$ & \\
\hline Severe & 4 & $(80.0)$ & 1 & $(20.0)$ & \\
\hline No pain & 141 & $(50.2)$ & 140 & $(49.8)$ & \\
\hline Not applicable & 23 & $(48.9)$ & 24 & $(51.1)$ & \\
\hline \multicolumn{6}{|l|}{ 8. Back } \\
\hline Mild & 43 & $(39.8)$ & 65 & $(60.2)$ & 0.047 \\
\hline Moderate & 32 & $(44.4)$ & 40 & $(55.6)$ & \\
\hline Severe & 3 & $(50.0)$ & 3 & $(50.0)$ & \\
\hline No pain & 116 & $(56.3)$ & 90 & $(43.7)$ & \\
\hline Not applicable & 21 & $(58.3)$ & 15 & $(41.7)$ & \\
\hline \multicolumn{6}{|l|}{ 9. Leg } \\
\hline Mild & 41 & (51.3) & 39 & $(48.7)$ & 0.029 \\
\hline
\end{tabular}




\begin{tabular}{|c|c|c|c|c|c|}
\hline \multirow[b]{2}{*}{ Moderate } & \multicolumn{2}{|c|}{ Male $(n=215)$} & \multicolumn{2}{|c|}{$\begin{array}{l}\text { Female }(n= \\
214)\end{array}$} & \\
\hline & 25 & (34.2) & 48 & $(65.8)$ & \\
\hline Severe & 7 & (38.9) & 11 & $(61.1)$ & \\
\hline No pain & 123 & $(54.9)$ & 101 & $(45.1)$ & \\
\hline Not applicable & 19 & $(55.9)$ & 15 & $(44.1)$ & \\
\hline \multicolumn{6}{|l|}{ 10. Knees } \\
\hline Mild & 46 & $(41.8)$ & 64 & $(58.2)$ & 0.008 \\
\hline Moderate & 31 & $(38.8)$ & 49 & $(61.2)$ & \\
\hline Severe & 11 & $(47.8)$ & 12 & $(52.2)$ & \\
\hline No pain & 110 & $(58.5)$ & 78 & $(41.5)$ & \\
\hline Not applicable & 17 & $(60.7)$ & 11 & $(39.3)$ & \\
\hline \multicolumn{6}{|l|}{ 11. Ankle } \\
\hline Mild & 27 & $(49.1)$ & 28 & $(50.9)$ & 0.265 \\
\hline Moderate & 15 & $(34.9)$ & 28 & $(65.1)$ & \\
\hline Severe & 5 & $(41.7)$ & 7 & $(58.3)$ & \\
\hline No pain & 147 & $(52.9)$ & 131 & $(47.1)$ & \\
\hline Not applicable & 21 & $(51.2)$ & 20 & $(48.8)$ & \\
\hline \multicolumn{6}{|l|}{ 12. Toes } \\
\hline Mild & 22 & $(42.3)$ & 30 & $(57.7)$ & 0.673 \\
\hline Moderate & 11 & $(42.3)$ & 15 & $(57.7)$ & \\
\hline Severe & 3 & $(50.0)$ & 3 & $(50.0)$ & \\
\hline No pain & 157 & $(52.0)$ & 145 & $(48.0)$ & \\
\hline Not applicable & 22 & $(51.2)$ & 21 & $(48.8)$ & \\
\hline $\begin{array}{l}n=\text { frequency } \\
a=\text { Chi-square test for independence }\end{array}$ & & & & & \\
\hline
\end{tabular}

Table 6 demonstrates the correlates of perceived health and satisfaction with life of the sample. For male participants, after adjusting for possible confounding factors, it was observed that number of children, estimated income, having chronic illness and diabetes were significantly associated with overall perceived health, explaining $33.1 \%$ of the total variance. Increase in number of children was observed to 
increase perception of overall health. Conversely, increase in income, chronic illness and diabetes were observed to reduce perception of overall health. In contrast, for female participants, $38.9 \%$ of the variance of overall perceived health could be explained by satisfaction with life and difficulty to do tasks that were normally easy. 
Factors associated with perceived health among elderly by gender $(n=429)$.
Male $(n=215)$
Female $(n=214)$

Adjusted b $((95 \% \mathrm{Cl}) \quad P$ value $\quad$ Adjusted b $((95 \% \mathrm{Cl}) \quad P$ value

Final model

Final model

\section{Perceived health}

No. of children

\begin{tabular}{|c|c|c|c|c|c|c|}
\hline 1 to 2 children & - & - & - & - & - & - \\
\hline 3 to 4 children & 0.79 & $(0.13,1.46)$ & 0.019 & - & - & - \\
\hline 5 to 6 children & 0.69 & $(0.04,1.35)$ & 0.038 & - & - & - \\
\hline$>6$ children & - & - & - & - & - & - \\
\hline Estimated income & -0.09 & $(-0.17,-0.02)$ & 0.013 & - & - & - \\
\hline Diagnosed with chronic illness & -0.69 & $(-1.03,-0.34)$ & $<0.001$ & - & - & - \\
\hline Health problem (diabetes) & -0.98 & $(-1.58,-0.38)$ & 0.002 & - & - & - \\
\hline Satisfaction with life & - & - & - & 0.32 & $(0.16,0.48)$ & $<0.001$ \\
\hline $\begin{array}{l}\text { Something that is normally } \\
\text { effortless was difficult to do }\end{array}$ & - & - & - & -0.25 & $(-0.46,-0.04)$ & 0.018 \\
\hline \multicolumn{7}{|l|}{ 2. Satisfaction with life } \\
\hline Difficulty to shower by self & -1.42 & $(-2.48,-0.37)$ & 0.009 & - & - & - \\
\hline Smoking & 0.29 & $(0.06,0.52)$ & 0.015 & - & - & - \\
\hline Concern about health & -0.19 & $(-0.30,-0.08)$ & 0.001 & - & - & - \\
\hline $\begin{array}{l}\text { Concern towards friends, } \\
\text { acquaintances or neighbours }\end{array}$ & 0.25 & $(0.03,0.48)$ & 0.028 & 0.30 & $(0.02,0.58)$ & 0.035 \\
\hline Walking in daily activities & -0.10 & $(-0.19,-0.01)$ & 0.028 & - & - & - \\
\hline Leg pain & -0.10 & $(-0.21,-0.00)$ & 0.046 & - & - & - \\
\hline Health problem (kidney) & -0.39 & $(-0.75,-0.03)$ & 0.034 & - & - & - \\
\hline Education level & - & - & - & -0.08 & $(-0.17,-0.00)$ & 0.044 \\
\hline Overall Perceived health & - & - & - & 0.43 & $(0.22,0.64)$ & $<0.001$ \\
\hline Concern towards spouse & - & - & - & -0.38 & $(-0.63,-0.14)$ & 0.003 \\
\hline
\end{tabular}


Multiple linear regression model

$b=$ Regression coefficient; $n=$ Frequency, $\mathrm{Cl}=$ Confidence interval

Multiple regression was also applied for satisfaction with life. For female participants, $45.6 \%$ of the variance could be explained by having concern for friends, acquaintances or neighbours, education level, perceived health, and concern towards and from spouse, after adjusting for possible confounding factors. It was observed that higher concern for friends, acquaintances or neighbours, perceived health, and concern from spouse, increases satisfaction with life among female participants. In contrast, $26.3 \%$ of the variance for satisfaction with life among male participants could be explained by difficulty to have shower by themselves, smoking, being concern about health, having concern for friends, acquaintances or neighbours, walking as part of daily activity, leg pain and kidney problem. It was observed that smoking and having concern for friends, acquaintances or neighbours increases satisfaction with life among male participants.

\section{Discussion}

This paper discovered several important findings on gender differences among elderly person in Brunei where aging is rapidly comparable to Japan and Korea $(24,25)$. First of all, elderly women generally show lower life satisfaction as they are not only susceptible to chronic illnesses and disabilities, but also receptive of burden associated with traditional female roles (26). This is consistent, in the present study and previous studies $(18,19)$, where elderly women reported significantly lower life satisfaction than men. However, there is contradictory results revealed in several countries. In South Korea, elderly men reported lower life satisfaction than women (20). In China, elderly women reported higher life satisfaction (21). In Spain, life satisfaction is equally shared between men and women (3).

Life satisfaction is a complex, multi-dimensional phenomenon, and the differences based on gender could possibly be mediated by other predictors such as socioeconomic and sociocultural factors on gender roles, opportunities and disadvantages across life events (26). In the present study, the highest predictor of life satisfaction among elderly women was positive self-perception of health, similar to a study in Brazil (26), together with other significant social factors including having concern for spouse friends, acquaintances or neighbours. In addition, we also revealed that women were still actively engaged in traditional gender roles such as care of children, household chores, and care of older relatives by themselves. Education level could also play an important part, where those with lower level of education were significantly more satisfied, which is congruent to a study in China (27) but in contrast to a study in Spain where higher education level contributed to higher life satisfaction (3). For elderly men, there was overlapping predictors with women, however, major differences were variables more incline towards physical activities and abilities. The highest predictor of life dissatisfaction among men was 
difficulty to perform daily activities, particularly taking shower. In our results, men had significantly lower proportions of musculoskeletal pain or discomfort, and they generally were able to perform daily activities without assistance. This could explain why elderly men reported higher life satisfaction than female. In South Korea, elderly men reported lower level of physical health as well as lower social relationship, which had contributed to lower life satisfaction and increased mental health issues $(20,28)$.

Healthy aging is tightly intertwined with life satisfaction as shown in our results, similar to prior studies (29-31). This is particularly evident for elderly women, as discussed above. The proportion of musculoskeletal discomfort as well as mental health and emotional conditions were significantly higher among elderly women. The high correlation between physical activity on mental health could explain this relationship. Previous studies have determined effects of exercise on mental health $(32,33)$, and the current Coronavirus disease 2019 (COVID-19) pandemic might deteriorate the situation further with mandated social isolation and restrictions on public movement (33). The World Health Organization has recommended at least 150 minutes per week of moderate-intensity physical activity such as brisk walking and riding a bike, or at least 75 minutes per week of vigorous-intensity physical activity such as running or fast swimming, for adults including elderly (34). This include home-based physical activity such as aerobic exercises, bodyweight training, dance and active gaming to counter physical and mental side effects of COVID-19 regulations (35). Furthermore, social participation has also been a critical indicator of positive health effects on elderly population (5). In Japan, a gender stratified analysis revealed that social participation improved physical and mental health of elderly women more than men, and overall benefits were recorded for both gender compared to non-participation (5). Being active in various social activities such as sport groups, hobby clubs, gardening, recreational training, and so forth, have demonstrated positive effects in different elderly populations $(5,36,37)$.

Moreover, in the present study, having more children were perceived as healthier among elderly men. Even though numerous studies have considered the association between health and number of children, very few have examined gender differences. We could only postulate that, in this population, elderly men may place importance in intergenerational transfers and support such as practical help and financial aid, while elderly women on emotional support and care (1). Another reason might institute from culturally - what constitute healthy or successful aging - defined achievement. This could include pride of continuing the family line, as well as related benefits of self-esteem gained, economic and social security, that might outweigh burden of having fewer or no children such as continuing unwanted involvement in children's lives, prolonged mental aggravations, and continuing financial demands from children (9).

The results of this study should be interpreted within its limitations. Cross-sectional nature of this study limits prospective causal inferences, and the self-reported data collected is subject to recall and reporting bias. Even though we have investigated major domains of elderly life satisfaction and healthy aging, future studies should include other dimensions that are no less salient such as spiritual experiences and perception of death (38) as well as emerging components such as gerontechnology and digital health, to provide a comprehensive and holistic approach towards successful aging. 
In conclusion, this study has made large strides in understanding gender differences in life satisfaction and healthy aging, as well as providing epidemiologic estimates in socioeconomic status, physical health, mental and emotional conditions, and social health status for elderly men and women. However, more efforts from policymakers and related stakeholders of elderly population, are still needed to close gender gaps, by ensuring living spaces, transportation, and communication services as well as community and other projects that increases physical activity, social interaction and maintain relationships among elderly population.

\section{Abbreviations}

HRS

Health and Retirement Study

ELSA

English Longitudinal Survey on Aging

UCL

University College London

IFS

Institute for Fiscal Studies

SHARE

Survey on Health, Aging and Retirement in Europe

TILDA

Irish Longitudinal Study on Aging

KLoSA

Korean Longitudinal Study of Aging

JSTAR

Japanese Study of Aging and Retirement

VIF

Variance inflation factor

COVID-19

Coronavirus disease 2019

\section{Declarations}

\section{Ethics approval and consent to participate}

The research protocol of this study has been approved by the research ethics committee of Universiti Brunei Darussalam (UBD/OAVCR/UREC/Apr18-04). This study conforms with ethical standards to ensure safeguarding and protection human rights were sufficient according to declaration of Helsinki (23). Written informed consent was obtained prior to the study. 
All authors reviewed and approved the final version of this manuscript for journal publication.

\section{Availability of data and material}

The data is available upon reasonable request.

\section{Competing interests}

The authors have no conflict of interest to declare.

\section{Funding}

This study was funded by Universiti Brunei Darussalam (UBD/RSCH/1.10/FICBF(b)/2018/004)

\section{Authors' contributions}

KHAM: Conception and design of work, data acquisition, revise critically and final approval.

YMY: Conception and design of work, data acquisition, revise critically and final approval.

LS: Conception and design of work, revise critically and final approval.

CY: Conception and design of work, revise critically and final approval.

HAR: Data analysis, results interpretation, drafting of work, revise critically and final approval.

AT: Conception and design of work, revise critically and final approval.

All authors: Responsible for the entire manuscript.

\section{Acknowledgements}

The authors would like to express utmost appreciation and gratitude to all the participants for supporting this study.

\section{References}

1. Hämäläinen $\mathrm{H}$, Tanskanen $\mathrm{AO}$. 'Sandwich generation': generational transfers towards adult children and elderly parents. J Fam Stud. 2019;

2. Sato Y, Yanagita M. The unprecedented era of aging. Inflamm Regen [Internet]. 2019;39(1):15. Available from: https://doi.org/10.1186/s41232-019-0104-2

3. Matud MP, García MC, Fortes D. Relevance of gender and social support in self-rated health and life satisfaction in elderly Spanish people. Int J Environ Res Public Health. 2019;16(15).

4. Kahn JR, McGill BS, Bianchi SM. Help to family and friends: Are there gender differences at older ages? J Marriage Fam. 2011;73(1):77-92. 
5. Tomioka K, Kurumatani N, Hosoi H. Positive and negative influences of social participation on physical and mental health among community-dwelling elderly aged 65-70 years: a cross-sectional study in Japan. BMC Geriatr [Internet]. 2017;17(1):111. Available from: https://doi.org/10.1186/s12877-017-0502-8

6. Tomioka $\mathrm{K}$, Kurumatani $\mathrm{N}$, Hosoi $\mathrm{H}$. Age and gender differences in the association between social participation and instrumental activities of daily living among community-dwelling elderly. BMC Geriatr. 2017;17(1):1-10.

7. Corrao S, Santalucia P, Argano C, Djade CD, Barone E, Tettamanti M, et al. Gender-differences in disease distribution and outcome in hospitalized elderly: Data from the REPOSI study. Eur J Intern Med [Internet]. 2014;25(7):617-23. Available from: http://dx.doi.org/10.1016/j.ejim.2014.06.027

8. Kim E-J, Jung S-W, Kim Y-E, Go D-S, Yoon S-J. Assessing the Impact of Aging on Burden of Disease. Iran J Public Health [Internet]. 2018 Jul;47(Suppl 1):33-8. Available from: https://pubmed.ncbi.nlm.nih.gov/30186810

9. Bocci C, Salvini S. Elderly with and without Children: Do They Report Different Health Conditions? In: ASA CONFERENCE 2019 Statistics for Health and Well-being BOOK OF SHORT PAPERS. 2019.

10. Kämpfen F, Wijemunige N, Evangelista B. Aging, non-communicable diseases, and old-age disability in low- and middle-income countries: a challenge for global health. Int J Public Health [Internet]. 2018;63(9):1011-2. Available from: https://doi.org/10.1007/s00038-018-1137-z

11. Beutell N. Life Satisfaction. In: Work and Family Encyclopedia. 2006.

12. Steptoe A, Deaton A, Stone AA. Subjective wellbeing, health, and ageing. Lancet. 2015 Feb;385(9968):640-8.

13. Blanchflower DG, Oswald AJ. The U-shape without controls: A response to Glenn. Soc Sci Med. 2009 Aug;69(4):486-8.

14. Blanchflower DG, Oswald AJ. Is well-being U-shaped over the life cycle? Soc Sci Med. 2008 Apr;66(8):1733-49.

15. Blanchflower DG, Oswald AJ. Well-being over time in Britain and the USA. J Public Econ. 2004 Jul;88(7-8):1359-86.

16. Baird BM, Lucas RE, Donnellan MB. Life Satisfaction Across the Lifespan: Findings from Two Nationally Representative Panel Studies. Soc Indic Res. 2010 Nov;99(2):183-203.

17. Puvill T, Lindenberg J, de Craen AJM, Slaets JPJ, Westendorp RGJ. Impact of physical and mental health on life satisfaction in old age: a population based observational study. BMC Geriatr. 2016 Dec;16(1):194.

18. Jovanović V, Joshanloo M. The determinants of life satisfaction in Serbia: Findings from the Gallup World Poll. Int J Wellbeing. 2019;9(1):43-60.

19. George LK. Still Happy After All These Years: Research Frontiers on Subjective Well-being in Later Life. Journals Gerontol Ser B [Internet]. 2010 May 1;65B(3):331-9. Available from: https://doi.org/10.1093/geronb/gbq006 
20. Park JH, Min S, Eoh Y, Park SH. The elderly living in single-person households in South Korea: a latent profile analysis of self-esteem, life satisfaction, and depression. Qual Life Res [Internet]. 2020; Available from: https://doi.org/10.1007/s11136-020-02693-1

21. Ng ST, Tey NP, Asadullah MN. What matters for life satisfaction among the oldest-old? Evidence from China. Quinn TJ, editor. PLoS One. 2017 Feb;12(2):e0171799.

22. United Nations, Department of Economic and Social Affairs PD. World Population Ageing 2019. World Population Ageing 2019 Highlights. United Nation; 2019. 5-11 p.

23. Williams JR. The Declaration of Helsinki and public health. Bull World Health Organ. 2008;86:650-2.

24. Othman A. Brunei to exceed Japan in ageing population by 2065. Borneo Bulletin. 2020;

25. Lovanxay B. ICT as one of the critical success factors of aging society in ASEAN: role and cooperation of Japan. In: Proceedings of the 8th International Conference on Theory and Practice of Electronic Governance. 2014. p. 510-1.

26. Pinto JM, Fontaine AM, Neri AL. The influence of physical and mental health on life satisfaction is mediated by self-rated health: A study with Brazilian elderly. Arch Gerontol Geriatr. 2016;65:104-10.

27. Pan Y, Chan SHW, Xu Y, Yeung KC. Determinants of life satisfaction and self-perception of ageing among elderly people in China: An exploratory study in comparison between physical and social functioning. Arch Gerontol Geriatr. 2019;84:103910.

28. Kang J-P, Yoon J. A Study on Multi-level Models in life satisfaction of Elderly Living alone: Moderating Effect of Elderly's Leisure Activity and Social Support. J Digit Converg. 2017;15(5):8998.

29. Ghimire S, Baral BK, Karmacharya I, Callahan K, Mishra SR. Life satisfaction among elderly patients in Nepal: associations with nutritional and mental well-being. Health Qual Life Outcomes. 2018;16(1):118.

30. Mehr MM, Zamani-Alavijeh F, Hasanzadeh A, Fasihi T. Effect of healthy lifestyle educational programs on happiness and life satisfaction in the elderly: A randomized controlled trial study. Iran J Ageing. 2019;13(4):440-51.

31. Park C-S, Park S-Y. Life satisfaction of elderly living alone based on their physical, mental and subjective oral health status. Korean J Heal Serv Manag. 2016;10(2):167-77.

32. Battaglia G, Bellafiore M, Alesi M, Paoli A, Bianco A, Palma A. Effects of an adapted physical activity program on psychophysical health in elderly women. Clin Interv Aging [Internet]. 2016 Jul 29;11:1009-15. Available from: https://pubmed.ncbi.nlm.nih.gov/27536081

33. Callow DD, Arnold-Nedimala NA, Jordan LS, Pena GS, Won J, Woodard JL, et al. The Mental Health Benefits of Physical Activity in Older Adults Survive the COVID-19 Pandemic. Am J Geriatr Psychiatry [Internet]. 2020 Oct 1;28(10):1046-57. Available from: https://doi.org/10.1016/j.jagp.2020.06.024

34. World Health Organization. \#HealthyAtHome - Physical activity. 2020.

35. Hammami A, Harrabi B, Mohr M, Krustrup P. Physical activity and coronavirus disease 2019 (COVID19): specific recommendations for home-based physical training. Manag Sport Leis [Internet]. 2020 
Apr 20;1-6. Available from: https://doi.org/10.1080/23750472.2020.1757494

36. Sanches A, Guzzoni V, Miranda VC dos R, Peressim LB, Rocha S, de Lima PO, et al. Recreational training improves cardiovascular adaptations, metabolic profile and mental health of elderly women with type-2 diabetes mellitus. Health Care Women Int. 2020;1-19.

37. Park S-A, Lee A-Y, Son K-C, Lee W-L, Kim D-S. Gardening intervention for physical and psychological health benefits in elderly women at community centers. Horttechnology. 2016;26(4):474-83.

38. Taghiabadi M, Kavosi A, Mirhafez SR, Keshvari M, Mehrabi T. The association between death anxiety with spiritual experiences and life satisfaction in elderly people. Electron physician [Internet]. 2017 Mar 25;9(3):3980-5. Available from: https://pubmed.ncbi.nlm.nih.gov/28461873 